\title{
Preoperative brachytherapy followed by laparoscopic hysterectomy: a new option to consider for early stages cervical cancer in the light of the LACC trial results
}

\author{
Clémence Beyer ${ }^{1}$, Houssein EL HAJJ ${ }^{2}$, Laurence Gonzague ${ }^{2}$, Leonel Varela Cagetti ${ }^{2}$, \\ Camille Jauffret $^{2}$, Guillaume Blache ${ }^{2}$, Laura Sabiani ${ }^{2}$, Gilles Houvenaeghel ${ }^{2}$, Magalie \\ Provansal $^{2}$, Renaud Sabatier ${ }^{2}$, and Eric Lambaudie ${ }^{2}$ \\ ${ }^{1}$ Grenoble Alpes University Hospital Centre Gynecology Department \\ ${ }^{2}$ Paoli-Calmettes Institute
}

May 11, 2020

\begin{abstract}
OBJECTIVES This study aims to report the outcomes of the combination of preoperative brachytherapy (POBT) followed by a Querleu Morrow Type A hysterectomy as an alternative to upfront surgery for early stage cervical cancer (ESCC) (2018 FIGO IA1-IB2). METHODS A single center retrospective study conducted between 2001 and 2012. After confirming the absence of pelvic node metastasis, all patients with ESCC underwent Low Dose Rate (LDR) POBT followed by Type A hysterectomy. Primary and secondary endpoints were the Disease Free Survival (DFS) and the associated morbidity respectively. RESULTS Out of the 138 patients included, complete response was found in $49.3 \%$ and a residual tumor $<1 \mathrm{~cm}$ in $26 \%$. After a median follow up of 132 months, DFS was $93.5 \%$ and 9 recurrences occurred. In univariate analysis, we found that delaying surgery more than 52 days after POBT is associated with a significant decrease in DFS $(\mathrm{p}=0.004)$. Pathological complete response was associated with an increased DFS $(\mathrm{p}=0.03)$. The brachytherapy related rate of late complications was $17.3 \%(\mathrm{n}=24)$ and the surgery related urinary tract complications rate was $6.5 \%(n=9)$, with only 2 patients $(1.5 \%)$ presented grade 3 complications CONCLUSION The multimodal radio-surgical management of ESCC appears to be a reasonable alternative to upfront open radical hysterectomy particularly in patients with high risk ESCC $(<2 \mathrm{~cm}$ associated with negative prognostic factors or for tumors measuring between 2 and $4 \mathrm{~cm}$ ). This approach is associated with low complications rate and a reasonable rate of local recurrences.
\end{abstract}

\section{Hosted file}

BJOG-20-0891.docx available at https://authorea.com/users/320738/articles/450196preoperative-brachytherapy-followed-by-laparoscopic-hysterectomy-a-new-option-toconsider-for-early-stages-cervical-cancer-in-the-light-of-the-lacc-trial-results 